\title{
Efficacy of cypermethrin, deltamethrin and nimbicidine on fecundity and egg viability of Tribolium castaneum (Herbst) (Coleoptera: Tenebrionidae)
}

\author{
R. Khatun, W. Islam and K.A.M.S.H. Mondal \\ Institute of Biological Sciences, University of Rajshahi, Rajshahi, Bangladesh
}

\begin{abstract}
Fecundity and egg viability of Tribolium castaneum (Herbst) females were significantly $(\mathrm{P}<0.01)$ reduced by synthetic pyrethroids and an alkaloid containing insecticide nimbicidine. In control, the average number of eggs laid by each female/day was 12.18 . The lowest number of eggs laid by each female/day was noted as 4.38 when combining nimbicidine with two pyrethroids i.e., cypermethrin and deltamethrin. The percentage of egg hatching without insecticide treatment was $95 \%$. The lowest percent of egg hatching was 63.95 when the adult female was fed on flour medium treated with combined doses of cypermethrin, deltamethrin and nimbicidine. Among the treated insecticides, nimbicidine $(80.69 \%)$ proved effective in reducing the fertility than those of cypermethrin (86.05\%) and deltamethrin (88.52\%).
\end{abstract}

Key words: Tribolium, cypermethrin, deltamethrin, nimbicidine, fecundity, fertility

\section{Introduction}

Oviposition in Tribolium is influenced by different environmental factors including moisture, temperature (Park \& Frank, 1948), relative humidity (Holdaway, 1932), flour medium (Khalequzzaman et al., 1994) and conditioning of the medium by the beetles living in it (Mondal \& Port, 1985). A fertile female oviposits no eggs in the first 3 days but after that at an increasing rate upto 18 eggs per day (Ashford, 1970).

Oviposition in Tribolium is also reduced by insecticides (Taher \& Cutkomp, 1983, Rahman, 1992; Kamaruzzaman, 2000; Hasnat, 2003), quinones and botanicals (Rahman, 1992; Akhtar, 1997; Banu, 2004; Khanom, 2004).

Viability of eggs also depends on different environmental factors such as temperature and relative humidity (Haque \& Islam, 1978), food (Khan \& Mazid, 1985; Khalequzzaman et al., 1994) and conditioning of food (Mondal, 1987).

Some conventional insecticides (Taher \& Cutkomp, 1983; Amin, 2000; Hasnat, 2003), reduced the progeny production of Tribolium species.

Active ingredient, azadirachtin of neem plant exhibited profound effects on fertility of stored product insect pests including Tribolium spp. (Khanam, 2003).

However, there is meagre information on the effects of cypermethrin, deltamethrin and nimbicidine alone or in combinations on both fecundity and progeny production of $T$. castaneum. So, present study has been undertaken.

\section{Materials and methods Fecundity}

$T$. castaneum adults were mass reared on fresh flour medium in a glass jar kept in an incubator at $30^{\circ} \mathrm{C}$ temperature without light and humidity control. After pupation, the pupae were collected by sieving the flour with a 500 micrometer sieve and sexed by microscopic examination of the exogenital process of the female pupae (Halstead, 1963). The male and female pupae were kept in the petri dish $(9 \mathrm{~cm}$ diam.) and observed until the emergence of adults.

Ten days old adults of known sex were paired with one from each sex and twenty five such pairs were used for oviposition experiment. Each pair was kept in a glass vial $(50 \times 25 \mathrm{~mm})$ containing $1 \mathrm{~g}$ of food either treated or untreated (acetone only). The upper surface of vials were plugged with cotton wool. The vials were kept in an incubator at $30^{\circ} \mathrm{C}$. Eggs were collected after every three days by sieving the flour media over a period of 45 days. The fresh food was supplied to each petri dish after every five days to avoid the conditioning of given food by the beetle themselves (Mondal \& Port, 1985). The adults used in the experiments were all survivors - those adults which were alive and perfectly normal in external appearance on emergence from the pupae without any deformities (Ashford, 1970). The flour media treated with cypermethrin $(0.5 \mathrm{ppm})$, deltamethrin (1ppm) and nimbicidine (6 ppm) individually and their different combinations were also used in the 
experiments. The same procedure was maintained for each treatment.

\section{Egg viability}

The eggs laid by the first 15 pairs of $T$. castaneum from each treatment were selected for the source of eggs. Eggs were collected from $3^{\text {rd }}$ to $45^{\text {th }}$ days with equal interval of 3 days from introduction of each pair to the experimental tubes/vials. The collected eggs were then kept in separate petri dishes for each treatment and incubated at $30^{\circ} \mathrm{C}$ without light and humidity control.

Eggs were regularly observed with a microscope and hatched larvae were carefully separated using the method described by Mondal \& Parween (1997). The percent of viability was calculated on the basis of the total number of first instar larvae that hatched from the number of eggs used (Mondal, 1987).

\section{Results and Discussion Fecundity}

The results and statistical analyses are shown in Fig 1 and Table 1 . The results were analyzed by Analysis of Variance and Duncan's Multiple Range Test (DMRT).

Cypermethrin, deltamethrin and nimbicidine had significant $(P<0.001)$ effects on reducing the fecundity of $T$. castaneum in comparison to that of the control.

The reduction of laying eggs in both cypermethrin and deltamethrin treatments is similar to the results of previous workers who reported that reduced fecundity in Tribolium was due to the effects of sub-lethal doses of DDT (Loschiavo,
1955; Taher \& Cutkomp, 1983), pirimiphos-methyl (Mondal \& Port, 1985; Rahman, 1992; Kamaruzzaman, 2000), lindane (Taher \& Cutkomp, 1983) and deltamethrin (Hasnat, 2003).

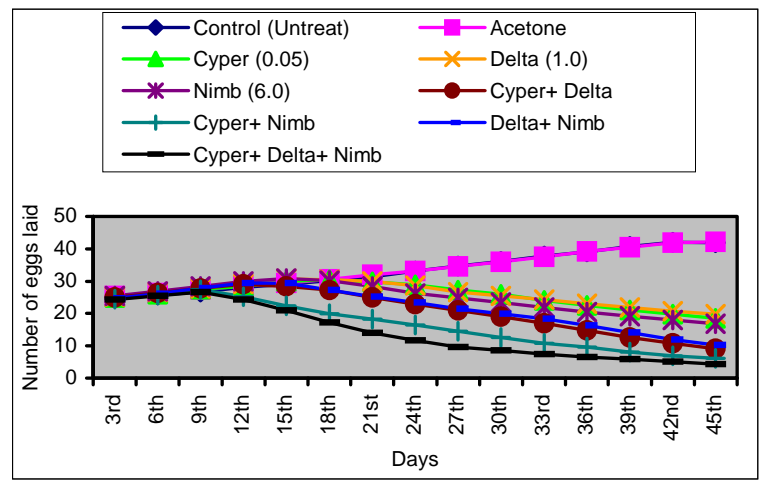

Fig 1. The average number of eggs laid by $T$. castaneum females at intervals of 3 days over a period of 45 days.

The reduction of fecundity due to nimbicidine found in the present experiment is similar to the findings of Banu (2004) who reported reduced fecundity in alkaloid, azadirachtin (technical grade $98 \%$ a.i) treatments on both $T$. castaneum and $T$. confusum. The present result is also similar to those of some previous workers who reported the reduction in oviposition of Tribolium due to botanicals (Amin, 2000; Khanam, 2003; Khanom, 2004). Azadirachtin incorporated into wheat flour reduced the fecundity significantly in both $T$. castaneum (Mukherjee \& Ramachandran (1989) and T. confusum (Hu \& Chiu, 1993)

Table 1. The average number of eggs laid by T. castaneum females reared on fresh medium (control) and medium treated with cypermethrin, deltamethrin and nimbicidine alone and their different combinations.

\begin{tabular}{|c|c|c|c|}
\hline \multirow{2}{*}{ Treatment (ppm) } & \multicolumn{3}{|c|}{ Eggs laid/day/female } \\
\hline & Mean SE & Minimum & Maximum \\
\hline Control (untreated) & $11.14+0.21^{\mathrm{a}}$ & 9.38 & 12.98 \\
\hline Acetone (As solvent) & $11.22+01^{\mathrm{a}}$ & 10.24 & 12.27 \\
\hline Cypermethrin (0.5ppm) & $8.55+0.09^{b}$ & 7.80 & 9.27 \\
\hline Deltamethrin (1.0ppm) & $8.64+0.06^{b}$ & 7.91 & 9.02 \\
\hline Nimbicidine (6.0ppm) & $8.24+0.05^{b}$ & 7.58 & 8.62 \\
\hline Cypermethrin (0.5)+Deltamethrin (1.0) & $7.03+0.07^{\mathrm{C}}$ & 6.62 & 7.73 \\
\hline Cypermethrin (0.5)+Nimbicidine (6.0) & $5.52+0.07^{d}$ & 5.18 & 6.27 \\
\hline Deltamethrin (1.0)+Nimbicidine (6.0) & $7.24+0.08^{c}$ & 6.47 & 7.71 \\
\hline Cypermethrin (0.5) + Deltamethrin (1.0) + & $4.71+0.04^{d}$ & 4.33 & 5.02 \\
\hline
\end{tabular}

Each experiment consists of 20 pairs (male: female $=1: 1$ ) and eggs collected at intervals of 3 days over a period of 45 days. Means followed by the same letters indicate no significant $(P>0.05)$ difference in the number of eggs laid by female adults (Duncan, 1955). 
It is evident that in both insecticides (synthetic pyrethroids) and nimbicidine (alkaloid) treatments, female laid few eggs at the beginning of the oviposition period and the numbers generally increased in course of time. But the rate of oviposition started to decrease after 30 days both in treatments and control (Fig 1). This might be due to prolonged period of feeding on treated medium affecting the metabolism of adult beetles which ultimately reduced the fecundity (Taher \& Cutkomp, 1983).

\section{Egg viability}

The results and statistical analyses are presented in Table 2. All the treatments significantly
$(P<0.001)$ reduced the fertility of eggs laid by $T$. castaneum females in comparison to those of the control.

The reduced rate of hatching in $T$. castaneum in the present experiment due to the insecticides cypermethrin and deltamethrin is similar to the findings of Mondal (1987). Rahman (1992) and Kamaruzzaman (2000) who reported significantly $(P<0.05)$ reduced fertility of Tribolium due to the effect of organophosphorus insecticide, pirimiphosmethyl. Taher \& Cutkomp (1983) also observed that both DDT and lindane significantly $(P<0.05)$ reduced fertility of $T$. confusum in comparison to control.

Table 2. The numbers and percentage to egg hatching in T. castaneum reared on fresh medium (control) and medium treated with cypermethrin, deltamethrin and nimbicidine alone and their different combinations.

\begin{tabular}{|c|c|c|c|}
\hline \multirow{2}{*}{ Treatment (ppm) } & \multirow{2}{*}{ Total Eggs Used } & \multicolumn{2}{|c|}{ Egg Hatching } \\
\hline & & Total Eggs Hatched & $\%$ Hatching \pm S.E \\
\hline Control (Untreated) & 2704 & 2669 & $95.0 \pm 0.30^{\mathrm{a}}$ \\
\hline Acetone (Control as solvents) & 2000 & 1721 & $94.75 \pm 0.34^{\mathrm{a}}$ \\
\hline Cypermethrin $(0.5)$ & 1960 & 1735 & $86.02 \pm 0.70^{b}$ \\
\hline Deltamethrin (1.0) & 1880 & 1517 & $88.52 \pm 0.56^{b}$ \\
\hline Nimbicidine (6.0) & 1485 & 1169 & $80.69+0.58^{b}$ \\
\hline Cypermethrin (0.5) + deltamethrin (1.0) & 1255 & 963 & $78.72+0.75^{c}$ \\
\hline Cypermethrin (0.5) + Nimbicidine (6.0) & 1645 & 1307 & $76.73 \pm 0.45^{c}$ \\
\hline Deltamethrin (1.0) + Nimbicidine (6.0) & 1082 & 692 & $79.73 \pm 0.55^{c}$ \\
\hline $\begin{array}{l}\text { Cypermethrin (0.5) + Deltamethrin (1.0) + } \\
\text { Nimbicidine (6.0) }\end{array}$ & 2573 & 2438 & $63.95 \pm 0.73^{d}$ \\
\hline
\end{tabular}

Each experiment consists of 20 pairs (male: female $=1: 1$ ) and eggs collected at intervals of 3 days over a period of 45 days. Figures followed by the same letters indicate no significant $(P>0.05)$ difference in percentage of egg hatching (Duncan, 1955).

The reduced rate of egg hatching due to nimbicidine (azadirachtin) found in the present experiment is similar to the findings of Khanam \& Talukder (1993) who reported the reduced fertility in $T$. castaneum and $T$. confusum due to botanicals like Bishkatali (Polygonum hydropiper $\mathrm{L})$, Neem (A. indica), Nishinda (Vitex negundo L.) and Royna (Aphanamixis polystachya W. \& A.). Both Neem leaf and seed extracts were also found to reduce the fertility of FSS-II and CTC-12 strains of $T$. castaneum significantly $(P<0.05)$ in comparison to that of control (Khanom, 2004). Banu (2004) reported that azadirachtin declined the fertility of both $T$. castaneum and $T$. confusum. The fertility of $T$. castaneum was minimized due to Neem plant extract treatment (Mannan et al., 1993).
The present result confirms the assertion that the fertility of eggs in Tribolium depends on environmental factors including food media. In the present experiment, the fertility was found to decline after 24 days exposure of $T$. castaneum to the treated media (Table 2). This might be due to longer period of feeding on treated medium affecting the metabolism of adult beetles and creating bad odours which ultimately reduced the fertility (Taher \& Cutkomp, 1983, Mondal, 1987). Depending on the stage of development oocytes develop abnormally and resorbed vitellogenesis is inhibited. Eggs laid by females fed on food medium treated with azadirachtin or neem extracts often have poorly formed chronic surfaces and may be more sensitive to timgal attack and are often less fertile (Schmutterer, 1987). 
According to (Loschiavo, 1955) chemical treatments probably affect the physiological state of the female which possibly induce a reduction in both fecundity and fertility. The net reduction of both fecundity and viability of eggs clearly demonstrates the potential means of reducing the population of $T$. castaneum.

\section{Acknowledgement}

The authors are grateful to $\mathrm{ACl}$ Bangladesh for supplying experimental samples of Cypermethrin, Deltamethrin and Nimbicidine and Institute of Biological Sciences, University of Rajshahi for laboratory facilities to conduct the experiment and providing financial support. The first author is grateful to the Ministry of Education, Government of the People's Republic of Bangladesh for granting deputation to pursue higher education.

\section{References}

Akhtar, N. 1997. Effectiveness of some plant oils against Tribolium castaneum (Herbst) and Tribolium confusum Duval (Coleoptera: Tenebrionidae). Unpublished PhD Thesis, University of Rajshahi, Bangladesh. 307pp.

Amin, T. 2000. Synergistic action of some indigenous plant materials with insecticides against Tribolium castaneum Herbst. Unpublished PhD Thesis, University of Rajshahi, Bangladesh. 255 pp.

Ashford, R.W. 1970. Some relationships between the red flour beetle, Tribolium castaneum (Herbst) (Coleoptera: Tenebrionidae) and Lymphotropha trioli Ashford (Neogregarinidae: Schizocystidae). Acta Protozool. 7:513-529.

Banu, M.J.A. 2004. Effects of azadirachtin on Tribolium castaneum and Tribolium confusum. Unpublished PhD Thesis, University of Rajshahi, Bangladesh. $324 \mathrm{pp}$.

Duncan, D.B. 1955. Multiple range and multiple F tests. Biometrics 11:1-41.

Halstead, D.G.H. 1963. External sex differences in stored-products Coleoptera. Bull. Ent. Res. 54:11934.

Haque, I. \& Islam, M.A. 1978. Effect of different food on the longevity and fecundity of Coccinella repanda Thumb. (Coleoptera: Coccinellidae). Bangladesh $\mathrm{J}$. Agric. Sci. 5(2):233-245.

Hasnat, H. 2003. Combined Action of Dimilin and Deltamethrin on Tribolium castaneum (Herbst). Unpublished Ph D Thesis, University of Rajshahi, Bangladesh. 273 pp.
Holdaway, F.G. 1932. An experimental study of the growth of populations of the flour beetle Tribolium castaneum Herbst as affected by atmospheric moisture. Ecol. Monogr. 2: 261-304.

$\mathrm{Hu}$, M.Y. \& Chiu, S.F. 1993. Experiments on the effectiveness of some botanical insecticides in controlling the confused flour beetle (Tribolium confusum). J. South-China Agric. Univ. 14: 4, 32-37.

Kamaruzzaman, A.H.M. 2000. Effects of cyromazine and pirimiphos-methyl on Tribolium castaenum Herbst and Tribolium confusum Duval. Unpublished PhD Thesis, University of Rajshahi, Bangladesh. 302 pp.

Khalequzzaman, M., Khanam, L.A.M. \& Talukdar, D. 1994. Growth of Tribolium confusum Duval on wheat flour with various yeast levels. Int. Pest. Cont. 36: 128-130.

Khan, A.R. \& Mazid, A. 1985. The oviposition and fertility of the confused flour beetle, Tribolium confusum Duval (Coleoptera: Tenebrionidae) on barley and rice flour. Univ. J. Zool. Rajshahi Univ. 4:8-10.

Khanam, L.A.M. 2003. Toxicity of some indigenous plant materials against Tribolium spp. throughout ontogeny. Unpublished PhD Thesis, University of Rajshahi, Bangladesh. 300 pp.

Khanam, L.A.M. \& Talukder, D. 1993. Effect of biskhatali Polygonum hydropiper L leaf and royna Aphanamixis polystachya Wall (Parker) seed coat extract on the fecundity and fertility of Tribolium confusum Duval (Coleoptera: Tenebrionidae). Bangladesh J. Sci. Ind. Res. 28(3):49-55.

Khanom, N.P. 2004. Effect of neem plant extracts and insect growth regulators on Tribolium castaneum (Herbst). Unpublished PhD Thesis, University of Rajshahi, Bangladesh. 339 pp.

Loschiavo, S.R. 1955. Rates of oviposition of Tribolium confusum surviving exposure to residues of pp DDT. Can. Entomol. 87:246-249.

Mannan, A., Rahman, S.M. \& Hossain, A. 1993. Reproductive potential of Tribolium castaneum (Herbst) (Coleoptera: Tenebrionidae) feed on flour treated with some plant extract. Tribolium Inf. Bull. 33:90-94.

Mondal, K.A.M.S.H. 1987. Effect of methylquinone, aggregation pheromone and pirimiphos-methyl on fertility of Tribolium castaneum Herbst. Univ. J. Zool. Rajshahi Univ. 26:21-26.

Mondal, K.A.M.S.H. \& Parween, S. 1997. Laboratory culturing of flour beetles, Tribolium species. Tribolium Inform. Bull. 37:153-162. 
Mondal, K.A.M.S.H. \& Port, G.R. 1985. Effect of methylquinone, aggregation pheromone and pirimiphos-methyl on the fecundity of Tribolium castaneum Herbst (Coleoptera: Tenebrionidae). Proc. Fifth Nat. Zool. Conf. Bangladesh. pp. 45-53.

Mukherjee, S.N. \& Ramachandran, R. 1989. Effects of azadirachtin on the feeding, growth and development of Tribolium castaneum Herbst. (Coleoptera: Tenebrionidae). J. Appl. Ent. 10(2):145-149.

Park, T. \& Frank, M.B. 1948. The fecundity and development of the flour beetles, Tribolium confusum and Tribolium castaneum at three constant temperatures. Ecology 29:368-372.
Rahman, A.S.M.S. 1992. Combined action of pirimiphos-methyl, synthetic methylquinone and botanicals on Tribolium confusum Duval. Unpublished PhD Thesis, University of Rajshahi, Bangladesh. 232 pp.

Schmutterer, H. 1987. Insect growth-disrupting and fecundity-reducing ingredients from the neem and chinaberry trees. In CRC Handbook of Natural Pesticides, Vol. III, Insect Growth Regulators, Part B (Morgan ED and Mandara NB, eds). CRC Press, Boca Raton, Florida. pp. 119-170.

Taher, M. \& Cutkomp, L.K. 1983. Effects of sublethal doses of DDT and three other insecticides on Tribolium confusum Duval (Coleoptera: Tenebrionidae). J. Stored Prod. Res. 19:43-50. 